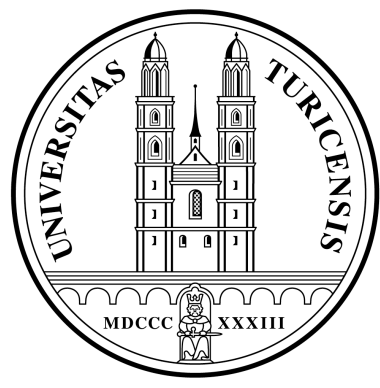

Institute for Empirical Research in Economics

University of Zurich

Working Paper Series

ISSN 1424-0459

Working Paper No. 91

Market Selection and Survival of Investment Strategies

Rabah Amir, Igor V. Evstigneev, Thorsten Hens, Klaus Reiner Schenk-Hoppé

October 2001 


\title{
Market Selection and Survival of Investment Strategies
}

\author{
Rabah Amir ${ }^{\mathrm{a}}$, Igor V. Evstigneev ${ }^{\mathrm{b}}$, Thorsten Hens ${ }^{\mathrm{c}}$ and \\ Klaus Reiner Schenk-Hoppé ${ }^{\mathrm{d}}$
}

\begin{abstract}
The paper analyzes the process of market selection of investment strategies in an incomplete asset market. The payoffs of the assets depend on random factors described in terms of a discrete-time Markov process. Market participants make dynamic investment decisions based on their observations and time. We show that a trader distributing wealth across available assets according to the relative expected returns eventually accumulates the entire market wealth. The result obtains under the assumption that the trader's strategy is asymptotically distinct from the CAPM strategy (prescribing investment in the market portfolio). This assumption turns out to be essentially necessary for the conclusion.
\end{abstract}

JEL-Classification: D52, D81, D83, G11.

Keywords: evolutionary finance, portfolio theory, investment strategies, CAPM, market selection, incomplete markets.

\section{Introduction}

The purpose of the paper is to develop an evolutionary approach to the study of investment strategies in financial markets. It has long been argued (Alchian 1950, Cootner 1964, Fama 1965) that market pressures would eventually select those traders who are better adapted to the prevailing conditions. According to the standard paradigm of economic theory, agents

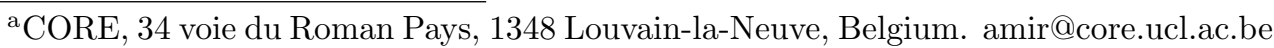

${ }^{\mathrm{b} S c h o o l ~ o f ~ E c o n o m i c ~ S t u d i e s, ~ U n i v e r s i t y ~ o f ~ M a n c h e s t e r, ~ O x f o r d ~ R o a d, ~ M a n c h e s t e r ~ M 13 ~}$ 9PL, UK. igor.evstigneev@man.ac.uk

${ }^{\mathrm{c}, \mathrm{d}}$ Institute for Empirical Research in Economics, University of Zurich, Blümlisalpstrasse 10, 8006 Zürich, Switzerland. thens@iew.unizh.ch, klaus@iew.unizh.ch
} 
maximize preferences or utilities. From the evolutionary point of view, what matters is not the utility level, but the chances of survival. The evolutionary principle leads to the consideration of the process of economic natural selection among the market participants (or among the strategies of behavior they adopt). This view, in comparison with the implications of utility or profit maximizing behavior, has been discussed in the context of financial market modelling by Blume and Easley (1992, 2001), Sandroni (2000), and others.

The approach pursued in the present work combines ideas from economic theory and finance. We examine the process of market selection in the framework of incomplete markets with traders that can dynamically update their investment strategies. While retaining a market-clearing mechanism that determines prices endogenously in every period, we depart from individual utility maximization. We assume that each trader follows a portfolio rule, specifying a distribution of wealth across the available assets for any moment of time and for any history of events. Trading strategies are compared with each other in terms of their abilities to survive under market selection in the long run (rather than in the conventional terms of discounted values). The model allows to include various types of agents' strategies, e.g., those motivated by behavioral finance (Shleifer 2000).

We analyze the global dynamics of the distribution of wealth across investors - each employing an individual portfolio rule -in a market with short-lived assets. The assets are in positive supply and their payoffs depend on the realization of an exogenous state of the world that is described in terms of a homogeneous finite-state Markov chain. Short sales are ruled out. In the case where only a complete set of Arrow securities is traded and the states of the world are independent and identically distributed (i.i.d.), our model reduces to the one proposed in the seminal paper by Blume and Easley (1992). We regard the model as being of interest from the applied perspective of financial markets as well as being theoretically sound.

The main result of our paper is that, in any - complete or incompletemarket for short-lived assets, there is one specific portfolio rule, denoted by $\lambda^{*}$, such that a trader following the rule eventually accumulates the entire market wealth. This result requires an asymptotic condition on the distance between the trader's strategy based on $\lambda^{*}$ and the CAPM rule. The latter prescribes investing into the market portfolio ${ }^{1}$. A trader using the CAPM rule keeps a constant fraction of the market wealth. Therefore she can neither accumulate the entire market wealth nor be driven out by the other investors.

\footnotetext{
${ }^{1}$ This investment rule is suggested by the Capital Asset Pricing Model (CAPM) and Tobin's mutual fund theorem-see, e.g., Magill and Quinzii (1996, Theorem 17.3 and Proposition 16.15).
} 
Investing into the market portfolio means mimicking the "average" portfolio. We prove that the $\lambda^{*}$-trader gathers market wealth at an exponential rate if $\lambda^{*}$ is bounded away from the CAPM rule for sufficiently many periods of time. More precisely, we impose the following condition: there exists a random number $\kappa>0$ such that, almost surely, the distance between $\lambda^{*}$ and the CAPM rule is greater than $\kappa$ in $n_{t}$ periods during every time-horizon of length $t$, where $\liminf _{t \rightarrow \infty} n_{t} / t>0$. Remarkably, this condition turns out to be not only sufficient but also necessary for the validity of the above assertion (see Theorem 3 in Section 3).

Our result shows that a $\lambda^{*}$-trader survives the market selection process regardless of the initial distribution of wealth among the traders. The portfolio rule $\lambda^{*}$ is of appealing simplicity: it is very easy to compute. The rule $\lambda^{*}$ requires a trader to distribute wealth across assets in accordance with the relative conditional expected returns of each asset. The result implies a simple valuation formula for the assets traded. In the limit, the price of each asset is determined by the risk-neutral valuation of its payoffs.

The principle underlying the rule $\lambda^{*}$ has been known and established in various contexts since the work of Kelly (1956) and Breiman (1961), whose studies were motivated, basically, by gambling models. This principle - in financial interpretation - prescribes that the investments should be proportional to the expected returns. If the outcomes are zero-one (win or lose), then the optimal strategy reduces to distributing investments according to the probabilities of the positive outcomes ("betting one's beliefs"). Kelly (1956) and Breiman (1961) have shown that this principle leads to the maximization of the expected logarithm of the growth rate. This idea gave rise to a large area of research - see, e.g., Thorp (1971), Algoet and Cover (1988), Hakansson and Ziemba (1995). Apparently, the important paper by Radner (1971) was the first where this idea was systematically developed in the context of economic theory. Radner applied it to the study of a stochastic analogue of the von Neumann-Gale model of economic growth (see, e.g., Arkin and Evstigneev (1987)).

Blume and Easley (1992) considered a model of a complete asset market with endogenous prices, simple (constant) trading strategies and i.i.d. states of the world. They have shown that, in order to be a single survivor in the market selection process, a trader should follow the rule of "betting one's beliefs." To find this rule it suffices to solve the maximization problem for the expected logarithm of a (properly defined) individual growth rate. Evstigneev, Hens, and Schenk-Hoppé (2001) extended the Blume-Easley approach to an incomplete market model with simple trading strategies and i.i.d. states of the world (see also Hens and Schenk-Hoppé (2001), where local dynamics of the process were studied). In that context, the problem 
of characterizing a single survivor cannot generally be reduced to individual optimization.

The present work continues this line of studies and makes progress in the following two directions. Firstly, we consider general, not necessarily simple, strategies, which substantially enlarges the area of applicability of the results. Secondly, we abandon the assumption of independence of the underlying random parameters. Rather, we assume that the underlying process, describing the states of the world, is Markov. Therefore our main result is formulated in terms of conditional (rather than unconditional) expectations of relative returns.

Although our result is complete within the present framework, there are certainly many desirable extensions of the model. One can mention, for instance, long-lived assets, changes in the market structure, endogenous asset supply, and variations of the investment-consumption ratio. These generalizations are left to future research.

The paper is organized as follows. Section 2 introduces the model. The main results are presented in Section 3. All the proofs are relegated to the Appendix.

\section{Model}

Let $S$ be a finite set and $s_{t}, t=0,1,2, \ldots$, a homogeneous Markov chain with transition function $p(\sigma \mid s)$, specifying the conditional probabilities $P\left\{s_{t+1}=\right.$ $\left.\sigma \mid s_{t}=s\right\}$. The random variable $s_{t}$ describes the "state of the world" at time $t$. There are $K$ assets that live one period but are identically reborn at every subsequent period. One unit of asset $k$ issued at time $t$ yields return $A_{k}\left(s_{t+1}, s_{t}\right) \geq 0$ at time $t+1$. We assume

$$
\sum_{k=1}^{K} A_{k}(\sigma, s)>0
$$

for all $\sigma, s \in S$.

There are $I$ investors (traders) $i=1, \ldots, I$ acting on the market. Every investor $i$ at each time $t=0,1,2, \ldots$ selects a portfolio

$$
h_{t}^{i}=\left(h_{1, t}^{i}, \ldots, h_{K, t}^{i}\right) \text {, }
$$

where $h_{k, t}^{i}$ is the number of units of asset $k$ in the portfolio $h_{t}^{i}$. Generally, $h_{t}^{i}$ depends on the history $s^{t}=\left(s_{0}, \ldots, s_{t}\right)$ of the process $s_{t}$ up to time $t$ :

$$
h_{t}^{i}=h_{t}^{i}\left(s^{t}\right)
$$


(we will often omit the argument $s^{t}$ when this does not lead to ambiguity). For each $t \geq 1, k$ and $s^{t}$, we have

$$
\sum_{i=1}^{I} h_{k, t}^{i}\left(s^{t}\right)=V_{k}\left(s_{t}\right)
$$

where $V_{k}\left(s_{t}\right)$ is the trading volume of asset $k$ at time $t \geq 0$ in the random situation $s_{t}$. The functions $V_{k}(s)>0$ of $s \in S(k=1, \ldots, K)$ are exogenously given in the model.

If investor $i$ possesses a portfolio $h_{t}^{i}=\left(h_{k, t}^{i}\right)$ at time $t \geq 0$, then her wealth $w_{t+1}^{i}$ at time $t+1$ can be expressed as

$$
w_{t+1}^{i}=\sum_{k=1}^{K} A_{k}\left(s_{t+1}, s_{t}\right) h_{k, t}^{i} .
$$

For every $i$, a strictly positive number $w_{0}^{i}$ is given-the initial wealth of investor $i$. In view of (2), we have

$$
\sum_{i=1}^{I} w_{t+1}^{i}=\sum_{k=1}^{K} A_{k}\left(s_{t+1}, s_{t}\right) V_{k}\left(s_{t}\right), \quad t \geq 0 .
$$

The variable

$$
w_{t}=\sum_{i=1}^{I} w_{t}^{i}
$$

specifies the aggregate market wealth at time $t \geq 0$.

It is assumed that every investor $i$ selects a portfolio by using the following procedure. He/she chooses an investment strategy - a sequence of functions

$$
\lambda_{t}^{i}=\left(\lambda_{1, t}^{i}, \ldots, \lambda_{K, t}^{i}\right), \lambda_{t}^{i}=\lambda_{t}^{i}\left(s^{t}\right), \quad t \geq 0,
$$

such that

$$
\lambda_{k, t}^{i}>0, \sum_{k=1}^{K} \lambda_{k, t}^{i}=1,
$$

and assigns the share $\lambda_{k, t}^{i}$ of her budget $w_{t}^{i}$ for purchasing asset $k$ at time $t$. Given every investor $i$ has chosen a strategy $\left(\lambda_{k, t}^{i}\right)$, the equation

$$
\rho_{k, t}=\frac{1}{V_{k}\left(s_{t}\right)} \sum_{i=1}^{I} \lambda_{k, t}^{i} w_{t}^{i}
$$


determines the market clearing price $\rho_{k, t}=\rho_{k, t}\left(s^{t}\right)$ of asset $k$ at any time $t \geq 0$. Then the portfolio $h_{t}^{i}$ of investor $i$ can be expressed as follows

$$
h_{k, t}^{i}=\frac{\lambda_{k, t}^{i} w_{t}^{i}}{\rho_{k, t}}, \quad t \geq 0
$$

From the last and the previous equations, we find

$$
h_{k, t}^{i}=V_{k}\left(s_{t}\right) \frac{\lambda_{k, t}^{i} w_{t}^{i}}{\sum_{j=1}^{I} \lambda_{k, t}^{j} w_{t}^{j}} .
$$

This leads to the following formula expressing the wealth $w_{t+1}^{i}$ of investors $i=1,2, \ldots, I$ at time $t+1$ through their wealth at time $t$ :

$$
w_{t+1}^{i}=\sum_{k=1}^{K} A_{k}\left(s_{t+1}, s_{t}\right) V_{k}\left(s_{t}\right) \frac{\lambda_{k, t}^{i} w_{t}^{i}}{\sum_{j=1}^{I} \lambda_{k, t}^{j} w_{t}^{j}} .
$$

Since $w_{0}^{i}>0$, we obtain by way of induction that $w_{t}^{i}>0$ for each $t$ (see (1) and (5)). From this we conclude that the evolution of the relative market shares of the investors,

$$
r_{t}^{i}=\frac{w_{t}^{i}}{w_{t}}
$$

is governed by the equations

$$
r_{t+1}^{i}=\sum_{k=1}^{K} R_{k}\left(s_{t+1}, s_{t}\right) \frac{\lambda_{k, t}^{i} r_{t}^{i}}{\sum_{j=1}^{I} \lambda_{k, t}^{j} r_{t}^{j}}, \quad i=1, \ldots, I,
$$

where

$$
R_{k}\left(s_{t+1}, s_{t}\right)=\frac{A_{k}\left(s_{t+1}, s_{t}\right) V_{k}\left(s_{t}\right)}{\sum_{m=1}^{K} A_{m}\left(s_{t+1}, s_{t}\right) V_{m}\left(s_{t}\right)} .
$$

The numbers $R_{k}\left(s_{t+1}, s_{t}\right)$ characterize the relative (normalized) payoffs of the assets $k=1,2, \ldots, K$. We have $R_{k}\left(s_{t+1}, s_{t}\right) \geq 0$ and

$$
\sum_{k=1}^{K} R_{k}\left(s_{t+1}, s_{t}\right)=1
$$

The main focus of this work is on the analysis of the evolution of the relative market shares $r_{t}^{i}$ depending on the choice of the strategies $\lambda_{t}^{i}, i=$ $1,2, \ldots, I$. We are interested primarily in those strategies which allow an investor to survive, i.e., to keep a positive relative market share in the limit, and, moreover, which allow the investor to dominate the market, i.e., to 
gather in the limit all the market wealth. A central role is played by the following notion. We say that an investor $i$ (or the strategy $\lambda^{i}=\left(\lambda_{k, t}^{i}\right)$ ) is a single survivor in the selection process (8) if

$$
\lim r_{t}^{i}=1
$$

almost surely (a.s.). Condition (10) implies $\lim r_{t}^{j}=0$ a.s. for all $j \neq i$, which means that, in the limit, investor $i$ accumulates all the market wealth. If the sequence $r_{t}^{i}$ involved in (10) converges to 1 at an exponential rate, we shall say that the strategy $\lambda^{i}$ dominates the others exponentially.

It is an important problem to identify those strategies which enable an investor using them to become a single survivor. Hens and Schenk-Hoppé (2001) and Evstigneev, Hens, and Schenk-Hoppé (2001) considered this problem within two different settings (local and global, respectively). The latter paper focused on a special case of the model at hand, where (i) the random variables $s_{t}$ are independent and identically distributed; (ii) the functions $A_{k}$ (and hence $R_{k}$ ) depend only on $s_{t+1}$; (iii) $V_{k}(s) \equiv 1$; and (iv) the analysis is restricted to the consideration of only simple strategies $\lambda^{i}=\left(\lambda_{k, t}^{i}\right)$, i.e., those for which the budget shares $\lambda_{k, t}^{i}\left(s^{t}\right)$ do not depend on $t$ and $s^{t}$. For that model, the following result was obtained (Evstigneev, Hens, and Schenk-Hoppé 2001, Theorem 1).

Theorem 1 Let the expected values $R_{k}^{*}=E R_{k}\left(s_{t}\right)$ be strictly positive and let the functions $R_{1}(s), \ldots, R_{K}(s)$ of $s \in S$ be linearly independent (the absence of redundant assets). Let one of the investors $i=1, \ldots, I$, say $i=1$, use the simple strategy $\lambda^{1}=\left(\lambda_{k}^{1}\right)$ defined by

$$
\lambda_{k}^{1}=R_{k}^{*}
$$

whereas all the other investors $i \neq 1$ use different simple strategies $\lambda^{i} \neq \lambda^{1}$. Then investor 1 is a single survivor in the market selection process (8).

This theorem generalizes the result of Blume and Easley (1992), dealing with the case of Arrow securities $\left(S=\{1,2, \ldots, K\}, A_{k}(s)=0\right.$ if $s \neq k$ and $A_{k}(s)=1$ if $s=k$ ). Furthermore, the strategy (11) defined in terms of the expected payoffs may be regarded as a development of the Kelly rule of "betting one's beliefs" (Kelly 1956). This rule was originally designed in connection with gambling problems, but later on it was successfully employed in portfolio theory (Thorp 1971, Aurell, Baviera, Hammarlid, Serva, and Vulpiani 2000).

In this work, we intend to obtain versions of Theorem 1 applicable to the more general model we have described in the present section. What is most 
essential in this generalization is that we are going to leave the framework of simple strategies and allow the investors to employ strategies using information about the history of the process $s_{t}$ - see the definition in (4) and (5). In this context, we can define a strategy $\lambda^{*}$ of "betting one's beliefs" — a direct analogue of the one considered in Theorem 1. As it turns out, we cannot, generally, guarantee $\lambda^{*}$ to be a single survivor. Nevertheless, we show that this conclusion does obtain under a natural sufficient condition, having a clear economic meaning. We also provide a necessary and sufficient condition for an investor using the strategy $\lambda^{*}$ to be a single survivor dominating the others exponentially. Precise statements of the results are given in the next section.

\section{Results}

Consider the random dynamical system (8) describing the evolution of the relative market shares $r_{t}^{i}\left(s^{t}\right)$ of the investors $i=1,2, \ldots, I$. Note that if $r_{t}=\left(r_{t}^{i}\right)$ is a strictly positive vector, then, as is easily seen from (5), (8) and (9), $r_{t+1}$ is a strictly positive vector as well. Thus $r_{t}=r_{t}\left(s^{t}\right)$ is a random process with values in the relative interior $\Delta_{+}^{I}$ of the unit simplex

$$
\Delta^{I}=\left\{x=\left(x^{1}, \ldots, x^{I}\right) \in \mathbb{R}^{I}: x^{i} \geq 0, \sum x^{i}=1\right\} .
$$

The initial state $r_{0}=\left(r_{0}^{1}, \ldots, r_{0}^{I}\right) \in \Delta_{+}^{I}$, from which this process starts, is fixed $\left(r_{0}^{i}=w_{0}^{i} / \sum w_{0}^{j}\right)$.

We will analyze the above random dynamical system under the following assumptions.

(A.1) The functions

$$
R_{k}^{*}(s):=\sum_{\sigma \in S} p(\sigma \mid s) R_{k}(\sigma, s), \quad k=1,2, \ldots, K,
$$

take on strictly positive values for each $s \in S$.

(A.2) For every $s \in S$, the functions $R_{1}(\cdot, s), \ldots, R_{K}(\cdot, s)$ restricted to the set

$$
\Pi(s)=\{\sigma \in S: p(\sigma \mid s)>0\}
$$

are linearly independent.

According to (A.1), the conditional expectation

$$
R_{k}^{*}(s)=E\left[R_{k}\left(s_{t+1}, s_{t}\right) \mid s_{t}=s\right]
$$


of the relative payoff $R_{k}\left(s_{t+1}, s_{t}\right)$ of every asset $k$ given $s_{t}=s$ is strictly positive at each state $s$. Hypothesis (A.2) means the absence of conditionally redundant assets. The term "conditionally" refers to the fact that the functions $R_{k}(\cdot, s), k=1, \ldots, K$, are linearly independent on the set $\Pi(s)$ - the support of the conditional distribution $p(\sigma \mid s)$.

In what follows, we will restrict attention to those investment strategies $\lambda=\left(\lambda_{k, t}\right)$ that satisfy the following additional assumption.

(B) The coordinates $\lambda_{k, t}\left(s^{t}\right)$ of the vectors $\lambda_{t}\left(s^{t}\right)$ are bounded away from zero by a strictly positive non-random constant $\rho$ (which might depend on the strategy $\lambda$, but not on $k, t$ and $s^{t}$ ).

In (5), we included in the definition of a strategy the condition $\lambda_{k, t}>0$ (such strategies are sometimes termed completely mixed). Assumption (B) contains the additional requirement of uniform strict positivity of $\lambda_{k, t}$.

A key role in our analysis will be played by the strategy $\lambda^{*}=\left(\lambda_{k, t}^{*}\left(s_{t}\right)\right)$ defined according to the formula

$$
\lambda_{k, t}^{*}\left(s_{t}\right)=R_{k}^{*}\left(s_{t}\right)
$$

where $R_{k}^{*}(s)$ is the conditional expectation of $R_{k}\left(s_{t+1}, s_{t}\right)$ given $s_{t}=s$ (see (12) and (13)). This is the strategy of "betting one's beliefs," which takes on, in the case of independent identically distributed variables $s_{t}$, the form (11). Note that $\lambda_{k}^{*}\left(s_{t}\right)=\lambda_{k, t}^{*}\left(s_{t}\right)$ does not explicitly depend on $t$, and, furthermore, $\lambda_{k}^{*}\left(s_{t}\right)$ is a function of only the current state $s_{t}$ of the process $\left(s_{t}\right)$, rather than the whole history $s^{t}$ of it. This implies, by virtue of (A.1) and in view of finiteness of $S$, that the strategy $\lambda^{*}$ satisfies condition (B).

To proceed further, we need to describe a recursive method of constructing strategies based on (Markovian) decision rules. Suppose one of the traders, say 1 , has a privilege of making her investment decision at time $t$ with full information about the current market structure $r_{t}$ and the actions $\lambda_{t}^{2}\left(s^{t}\right)$, $\lambda_{t}^{3}\left(s^{t}\right), \ldots, \lambda_{t}^{I}\left(s^{t}\right)$ that have just been undertaken by all the other traders $2,3, \ldots, I$. Formally, the decision of investor 1 is specified by a function

$$
f_{t}\left(r, l^{2}, \ldots, l^{I}\right), r \in \Delta_{+}^{I}, l^{j} \in \Delta_{+}^{K} \quad(j=2,3, \ldots, K)
$$

taking values in $\Delta_{+}^{K}$. Suppose such functions - decision rules - are given for all $t=0,1,2, \ldots$. Furthermore, suppose investors $2, \ldots, I$ have chosen some strategies $\lambda_{t}^{2}, \ldots, \lambda_{t}^{I}(t=0,1,2, \ldots)$. Then we can construct a strategy $\lambda_{t}^{1}\left(s^{t}\right)$, $t=0,1, \ldots$, of investor 1 by using the formula

$$
\lambda_{t}^{1}\left(s^{t}\right)=f_{t}\left(r_{t}, \lambda_{t}^{2}, \ldots, \lambda_{t}^{I}\right),
$$

where $r_{t}=r_{t}\left(s^{t}\right)$ and $\lambda_{t}^{j}=\lambda_{t}^{j}\left(s^{t}\right), j=2, \ldots, I$. 
Let us consider a particular decision rule $f=\left(f_{1}, \ldots, f_{K}\right)$ (which does not explicitly depend on $t$ ) defined by

$$
f\left(r, l^{2}, \ldots, l^{I}\right)=\sum_{j=2}^{I} \frac{r^{j}}{1-r^{1}} l^{j} .
$$

Here $r=\left(r^{1}, \ldots, r^{I}\right) \in \Delta_{+}^{I}, l^{j}=\left(l_{1}^{j}, \ldots, l_{K}^{j}\right) \in \Delta_{+}^{K}$, and so the vector $f=$ $\left(f_{1}, \ldots, f_{K}\right)$ belongs to $\Delta_{+}^{K}$. Note that the vector $f$ is a convex combination of the vectors $l^{2}, \ldots, l^{I}$ with weights $r^{j}\left(1-r^{1}\right)^{-1}$. This implies, in particular, the following: if the coordinates $l_{k}^{j}$ of the vectors $l^{j}$ are bounded away from 0 by a constant $\rho>0$, then the coordinates $f_{k}$ of $f$ are bounded away from 0 by the same constant. Consequently, if the strategies $\lambda_{t}^{2}, \ldots, \lambda_{t}^{I}$ satisfy condition (B), the strategy (15) satisfies condition (B) as well. In what follows, we will use the notation $f=\left(f_{k}\right)$ for the particular decision rule described in (16).

The decision rule (16) has a number of remarkable properties. First of all, observe the following. Suppose investor 1 employs the strategy $\lambda_{t}^{1}\left(s^{t}\right)$ defined by (15) in terms of the decision rule (16). Then we have

$$
\lambda_{k, t}^{1}=\sum_{j=1}^{I} \lambda_{k, t}^{j} r_{t}^{j}
$$

which, in view of $(8)$, yields

$$
r_{t+1}^{1}=r_{t}^{1} .
$$

Thus, if investor 1 uses the strategy generated by the decision rule (16), then (regardless of what strategies are used by the others!) the relative market share of this investor remains constant over time. This observation leads to the following conclusion. If one of the traders $2, \ldots, I$ uses the strategy $\lambda^{*}$, she cannot be a single survivor, as long as trader 1 uses the strategy (15) and, consequently, keeps a constant positive market share $r_{t}^{1}=r_{0}^{1}$ for all $t$.

Further, we can see that the portfolio of investor 1, who uses the strategy $\lambda_{t}^{1}$ defined in terms of the decision rule (16), is given by

$$
h_{k, t}^{1}=V_{k}\left(s_{t}\right) \frac{\lambda_{k, t}^{1}, w_{t}^{1}}{\sum_{j=1}^{I} \lambda_{k, t}^{j} w_{t}^{j}}=V_{k}\left(s_{t}\right) \frac{\lambda_{k, t}^{1} r_{t}^{1}}{\sum_{j=1}^{I} \lambda_{k, t}^{j} r_{t}^{j}}=V_{k}\left(s_{t}\right) r_{t}^{1},
$$

for all $k=1,2, \ldots, K($ see $(6)$ and $(17))$. Thus the vector $h_{t}^{1}=\left(h_{1, t}^{1}, \ldots, h_{K, t}^{1}\right)$ turns out to be proportional to the market portfolio, i.e., the vector

$$
\left(V_{1}\left(s_{t}\right), \ldots, V_{k}\left(s_{t}\right)\right)
$$


whose components indicate the amounts of assets $k=1,2, \ldots, K$ currently traded at the market. According to the well-known Tobin mutual fund theorem (Magill and Quinzii 1996, Proposition 16.15), portfolios having this structure result from the mean-variance optimization in the Capital Asset Pricing Model (CAPM). Therefore it is natural to term the decision rule (16) the CAPM decision rule and the strategy generated by it the CAPM strategy. The CAPM decision rule plays a key role in the formulation of the main results below.

In Theorem 2 below, we describe a condition sufficient for the strategy (14) to be a single survivor. We consider the dynamical system (8), assuming that the investors $i \in\{1,2, \ldots, I\}$ use some strategies $\lambda^{i}=\left(\lambda_{t}^{i}\right)$ satisfying requirement (B). We define

$$
\zeta_{t}=\left(\zeta_{1, t}, \ldots, \zeta_{K, t}\right)=f\left(r_{t}, \lambda_{t}^{2}, \ldots, \lambda_{t}^{I}\right)
$$

where $f$ is the CAPM decision rule. The symbol $|\cdot|$ denotes the sum of the absolute values of the coordinates of a finite-dimensional vector.

Theorem 2 Let investor 1 use the strategy $\lambda^{1}=\lambda^{*}$ defined by (14). Let the following condition be fulfilled:

(C) With probability 1, we have

$$
\liminf _{t \rightarrow \infty}\left|\lambda^{*}\left(s_{t}\right)-\zeta_{t}\right|>0 .
$$

Then investor 1 is a single survivor, and, moreover,

$$
\liminf _{t \rightarrow \infty} \frac{1}{t} \ln \frac{r_{t}^{1}}{1-r_{t}^{1}}>0
$$

almost surely.

Property (19) means that the relative market share of investor 1 tends to one at an exponential rate, whereas the relative market shares of all the other investors vanish at such rates, and so the strategy $\lambda^{*}$ dominates the others exponentially.

Condition $(\mathrm{C})$ can be restated as follows: there exists a strictly positive random variable $\kappa$ such that, almost surely,

$$
\left|\lambda^{*}\left(s_{t}\right)-\zeta_{t}\left(s^{t}\right)\right| \geq \kappa
$$

for all $t$ large enough. The last inequality requires that the actions $\lambda^{*}\left(s_{t}\right)$ prescribed by the strategy $\lambda^{*}$ should differ by not less than $\kappa>0$ from the actions

$$
\zeta_{t}\left(s^{t}\right)=\left(\zeta_{1, t}\left(s^{t}\right), \ldots, \zeta_{K, t}\left(s^{t}\right)\right), \quad \zeta_{k, t}\left(s^{t}\right)=\sum_{j=2}^{I} \frac{r_{t}^{j}\left(s^{t}\right)}{1-r_{t}^{1}\left(s^{t}\right)} \lambda_{k, t}^{j}\left(s^{t}\right),
$$


prescribed by the CAPM decision rule. Here, we do not assume that any of the market actors indeed employ the CAPM rule; we need it only as an indicator, a proper deviation of which from $\lambda^{*}$ guarantees $\lambda^{*}$ to be a single survivor.

In concrete instances, it might not be easy to verify condition (C) directly. Therefore we provide another hypothesis, (C.1), which is stronger than (C) but can conveniently be checked in various examples.

(C.1) There exists a strictly positive random variable $\kappa$ such that, with probability 1 , the distance between the vector $\lambda^{*}\left(s_{t}\right) \in \mathbb{R}^{K}$ and the convex hull of the vectors $\lambda_{t}^{2}\left(s^{t}\right), \ldots, \lambda_{t}^{I}\left(s^{t}\right) \in \mathbb{R}^{K}$ is not less than $\kappa$ for all $t$ large enough.

Clearly (C.1) implies (C) because $\zeta_{t}=f\left(r_{t}, \lambda_{t}^{2}, \ldots, \lambda_{t}^{I}\right)$ is a convex combination of $\lambda_{t}^{2}, \ldots, \lambda_{t}^{I}$.

Condition (C), which is sufficient for investor $i$ to be a single survivor, turns out to be close to a necessary one. The theorem below provides a version of hypothesis $(\mathrm{C})$ that is necessary and sufficient for the conclusion of Theorem 2 to hold.

Theorem 3 Investor 1 using the strategy (14) is a single survivor in the market selection process, and, moreover, dominates the others exponentially, if and only if the following condition is fulfilled:

(C.2) There exists a random variable $\kappa>0$ such that

$$
\liminf _{T \rightarrow \infty} \frac{1}{T} \#\left\{t \in\{0, \ldots, T\}:\left|\lambda^{*}\left(s_{t}\right)-\zeta_{t}\left(s^{t}\right)\right| \geq \kappa\right\}>0
$$

with probability 1.

The symbol \# in the above formula stands for the number of elements in a finite set.

Observe that (C.2) follows from (C). Indeed, (C) is equivalent to the existence of a random variable $\kappa$ for which, almost surely, inequality (20) is fulfilled for all $t$ large enough. In this case, the limit in (21) is equal to 1 . The limit in (21) may be thought of as a density (in the set of natural numbers) of those natural numbers $t$ for which inequality (20) holds. Hypothesis (C.2) only requires this density to be strictly positive, whereas (C) says that (20) should hold from some $t$ on.

Let us return to Theorem 2. From this theorem, it follows immediately that if the relation

$$
\liminf _{t \rightarrow \infty} \frac{1}{t} \ln \frac{r_{t}^{1}}{1-r_{t}^{1}} \leq 0
$$

holds with positive probability, then, with positive probability, there exists a (random) sequence $t_{k}$ such that

$$
\left|\lambda^{*}\left(s_{t_{k}}\right)-\zeta_{t_{k}}\left(s^{t_{k}}\right)\right| \rightarrow 0 .
$$


Can we make a stronger statement about convergence in (23) if we strengthen (22) appropriately? A result along these lines is provided by the next theorem.

Theorem 4 Let the following condition be satisfied:

(D.1) There exists a random variable $0<\gamma<1$ such that $E \ln \gamma>-\infty$ and

$$
r_{t}^{1}<1-\gamma
$$

a.s. for all $t$.

Then we have

$$
\left|\lambda^{*}\left(s_{t}\right)-\zeta_{t}\right| \rightarrow 0 \quad \text { a.s. }
$$

We will actually prove Theorem 4 under a weaker assumption:

(D.2) The expectations

$$
E\left[\ln \left(1-r_{T}^{1}\right)\right]
$$

do not converge to $-\infty$.

Clearly (D.1) is stronger than both (D.2) and (22), but (D.2) does not necessarily imply (22). Condition (D.1) holds, for example, if one of the investors $i=2, \ldots, I$ uses the CAPM strategy (and so her relative market share remains constant). Then, as Theorem 4 asserts, the difference between the budget shares of investor 1 prescribed by the strategy $\lambda^{*}$ and the budget shares prescribed by the CAPM decision rule converges a.s. to zero.

\section{Appendix}

\section{A.1 Proofs of the Main Results}

Theorem 2 is a direct consequence of Theorem 3 .

Proof of Theorem 3. By using (8), we write

$$
\begin{aligned}
\frac{1-r_{t+1}^{1}}{1-r_{t}^{1}} & =\frac{\sum_{i=2}^{I} r_{t+1}^{i}}{1-r_{t}^{1}} \\
& =\sum_{k=1}^{K} R_{k}\left(s_{t+1}, s_{t}\right) \frac{\left(1-r_{t}^{1}\right)^{-1} \sum_{i=2}^{I} \lambda_{k}^{i} r_{t}^{i}}{q_{k, t}}=\sum_{k=1}^{K} R_{k}\left(s_{t+1}, s_{t}\right) \frac{\zeta_{k, t}}{q_{k, t}}
\end{aligned}
$$

where

$$
q_{k, t}=\sum_{m=1}^{I} \lambda_{k}^{m} r_{t}^{m}=\lambda_{k}^{1} r_{t}^{1}+\left(1-r_{t}^{1}\right) \frac{\sum_{i=2}^{I} \lambda_{k}^{i} r_{t}^{i}}{1-r_{t}^{1}}=\lambda_{k}^{1} r_{t}^{1}+\zeta_{k, t}\left(1-r_{t}^{1}\right) .
$$


Consequently,

$$
1-r_{t+1}^{1}=\sum_{k=1}^{K} R_{k}\left(s_{t+1}, s_{t}\right) \frac{\zeta_{k, t}\left(1-r_{t}^{1}\right)}{\lambda_{k}^{1} r_{t}^{1}+\zeta_{k, t}\left(1-r_{t}^{1}\right)},
$$

and

$$
r_{t+1}^{1}=\sum_{k=1}^{K} R_{k}\left(s_{t+1}, s_{t}\right) \frac{\lambda_{k}^{1} r_{t}^{1}}{\lambda_{k}^{1} r_{t}^{1}+\zeta_{k, t}\left(1-r_{t}^{1}\right)} .
$$

For each $t=1,2, \ldots$, consider the random variable

$$
D_{t}=\ln \frac{r_{t}^{1}\left(r_{t-1}^{1}\right)^{-1}}{\left(1-r_{t}^{1}\right)\left(1-r_{t-1}^{1}\right)^{-1}} .
$$

We have

$$
D_{1}+\ldots+D_{T}=\ln \frac{r_{T}^{1}}{\left(1-r_{T}^{1}\right)}-\ln \frac{r_{0}^{1}}{\left(1-r_{0}^{1}\right)},
$$

Therefore, (19) holds if and only if

$$
\liminf _{T \rightarrow \infty} \frac{1}{T}\left(D_{1}+\ldots+D_{T}\right)>0 \quad \text { a.s. }
$$

By virtue of assumption (B), for every set of strategies $\left(\lambda_{k, t}^{i}\right), i=1, \ldots, I$, we consider, there exists a constant $H$ such that $\left(\min _{i, k} \lambda_{k}^{i}\right)^{-1} \leq H$. For this $H$, we have

$$
H^{-1} \leq \frac{r_{t+1}^{i}}{r_{t}^{i}} \leq H, \quad i=1, \ldots, I
$$

This implies

$$
H^{-1} \leq \frac{1-r_{t+1}^{1}}{1-r_{t}^{1}} \leq H
$$

because $1-r_{t}^{1}=\sum_{m=2}^{I} r_{t}^{m}$. Consequently, the random variables $D_{t}$ are uniformly bounded.

We have the following identity

$$
\frac{1}{T} \sum_{t=1}^{T} D_{t}=\frac{1}{T} \sum_{t=1}^{T} E\left(D_{t} \mid s^{t-1}\right)+\frac{1}{T} \sum_{t=1}^{T}\left[D_{t}-E\left(D_{t} \mid s^{t-1}\right)\right] .
$$

Since the random variables $D_{t}$ are uniformly bounded, we can apply to the process of martingale differences $B_{t}:=D_{t}-E\left(D_{t} \mid s^{t-1}\right)$ the strong law of 
large numbers (Hall and Heyde 1980, Theorem 2.19), which yields $T^{-1}\left(B_{1}\right.$ $\left.+\ldots+B_{T}\right) \rightarrow 0$ with probability 1 . Thus, we have

$$
\liminf _{T \rightarrow \infty} \frac{1}{T} \sum_{t=1}^{T} D_{t}=\liminf _{T \rightarrow \infty} \frac{1}{T} \sum_{t=1}^{T} E\left(D_{t} \mid s^{t-1}\right),
$$

and so (19) is equivalent to

$$
\liminf _{T \rightarrow \infty} \frac{1}{T} \sum_{t=1}^{T} E\left(D_{t} \mid s^{t-1}\right)>0 \quad \text { a.s. }
$$

By using (24), (25), we write

$$
\begin{aligned}
& E\left[D_{t} \mid s^{t-1}\right]=E\left[\ln \frac{r_{t}^{1}\left(r_{t-1}^{1}\right)^{-1}}{\left(1-r_{t}^{1}\right)\left(1-r_{t-1}^{1}\right)^{-1}} \mid s^{t-1}\right] \\
& =\sum_{\sigma \in S} p\left(\sigma \mid s_{t-1}\right) \ln \frac{\sum_{k} R_{k}\left(\sigma, s_{t-1}\right) \frac{\lambda_{k, t-1}^{1}}{\lambda_{k, t-1}^{1} r_{t-1}^{1}+\zeta_{k, t-1}\left(1-r_{t-1}^{1}\right)}}{\sum_{k} R_{k}\left(\sigma, s_{t-1}\right) \frac{\zeta_{k, t-1}}{\lambda_{k, t-1}^{1} r_{t-1}^{1}+\zeta_{k, t-1}\left(1-r_{t-1}^{1}\right)}}
\end{aligned}
$$

where

$$
\begin{gathered}
\zeta_{k, t-1}=\zeta_{k, t-1}\left(s^{t-1}\right)=\frac{\sum_{i=2}^{I} \lambda_{k, t-1}^{i} r_{t-1}^{i}}{1-r_{t-1}^{1}}, \\
\lambda_{k, t-1}^{i}=\lambda_{k, t-1}^{i}\left(s^{t-1}\right), r_{t-1}^{i}=r_{t-1}^{i}\left(s^{t-1}\right), \\
\lambda_{k, t-1}^{1}=\lambda_{k, t-1}^{1}\left(s_{t-1}\right)=R_{k}^{*}\left(s_{t-1}\right) .
\end{gathered}
$$

Let us use Lemma 1 (see Section A.2 below) to estimate the expression in (29). In view of this lemma, we have

$$
E\left(D_{t} \mid s^{t-1}\right) \geq \delta_{\rho}\left(\left|R^{*}\left(s_{t-1}\right)-\zeta_{t-1}\left(s^{t-1}\right)\right|\right),
$$

where $\rho$ is the strictly positive constant bounding away from zero the coordinates of $\lambda_{t}^{i}$. Denote by $N(T)=N\left(T, s^{T}\right)$ the set of those $t \in[0, T]$ for which $\left|R^{*}\left(s_{t}\right)-\zeta_{t}\left(s^{t}\right)\right| \geq \kappa$. We have

$$
\begin{aligned}
& \liminf _{T \rightarrow \infty} \frac{1}{T} \sum_{t=1}^{T} E\left(D_{t} \mid s^{t-1}\right) \geq \liminf _{T \rightarrow \infty} \frac{1}{T} \sum_{t=1}^{T} \delta_{\rho}\left(\left|R^{*}\left(s_{t-1}\right)-\zeta_{t-1}\left(s^{t-1}\right)\right|\right) \\
& \quad \geq \liminf _{T \rightarrow \infty} \frac{1}{T} \sum_{t=0}^{T-1} \delta_{\rho}\left(\left|R^{*}\left(s_{t}\right)-\zeta_{t}\left(s^{t}\right)\right|\right) \geq \liminf _{T \rightarrow \infty} \frac{1}{T} \sum_{t \in N(T-1)} \delta_{\rho}\left(\left|R^{*}\left(s_{t}\right)-\zeta_{t}\left(s^{t}\right)\right|\right) \\
& \geq \delta_{\rho}(\kappa) \cdot \liminf _{T \rightarrow \infty} \frac{1}{T} \#\{N(T-1)\}>0,
\end{aligned}
$$


where the last inequality follows from (C.2). Thus we have established (28), which is equivalent to (19).

Now, suppose that (19), and hence (28), hold. By virtue of Lemma 1, we find

$$
E\left(D_{t} \mid s^{t-1}\right) \leq L_{\rho} \cdot\left|R^{*}\left(s_{t-1}\right)-\zeta_{t-1}\left(s^{t-1}\right)\right|,
$$

and so (28) yields

$$
\liminf _{T \rightarrow \infty} \frac{1}{T} \sum_{t=1}^{T} d_{t}>0 \quad \text { a.s. }
$$

where $d_{t}=\left|R^{*}\left(s_{t-1}\right)-\zeta_{t-1}\left(s^{t-1}\right)\right|$.

Denote by $\bar{\kappa}$ the strictly positive random variable which is equal a.s. to the $\lim \inf$ in (33) and set $\kappa=\bar{\kappa} / 2$. We claim that

$$
\liminf \frac{1}{T} \#\left\{t \in\{1, \ldots, T\}: d_{t} \geq \kappa\right\}>0,
$$

which is equivalent to (C.2). Indeed, suppose the contrary. Then there is a sequence $T_{k}$ such that

$$
\frac{1}{T_{k}} \#\left\{t \in\left\{1, \ldots, T_{k}\right\}: d_{t} \geq \kappa\right\} \rightarrow 0 .
$$

For each $k$ denote by $M_{k}$ (resp. $N_{k}$ ) the set of those $t \in\left\{1, T_{k}\right\}$ for which $d_{t} \geq \kappa\left(\right.$ resp. $\left.d_{t}<\kappa\right)$. Then we have

$$
\frac{1}{T_{k}} \sum_{t=1}^{T_{k}} d_{t}=\frac{1}{T_{k}} \sum_{t \in M_{k}} d_{t}+\frac{1}{T_{k}} \sum_{t \in N_{k}} d_{t} \leq 2 \cdot \frac{1}{T_{k}} \#\left(M_{k}\right)+\kappa
$$

because $d_{t} \leq 2$. According to $(35),\left(T_{k}\right)^{-1} \cdot \#\left(M_{k}\right) \rightarrow 0$. Consequently,

$$
\liminf \frac{1}{T_{k}} \sum_{t=1}^{T_{k}} d_{t} \leq \kappa<\bar{\kappa},
$$

which contradicts the definition of $\bar{\kappa}$.

Proof of Theorem 4. Consider the nonnegative random variables $v_{t}=$ $\delta_{\rho}\left(\left|R^{*}\left(s_{t-1}\right)-\zeta_{t-1}\left(s^{t-1}\right)\right|\right)$. By using (32), we write $E v_{t} \leq E\left[E\left(D_{t} \mid s^{t-1}\right)\right]=$ $E D_{t}$, which yields, in view of (26),

$$
\sum_{t=1}^{T} E v_{t} \leq E \ln \frac{r^{1}}{1-r_{T}^{1}}+C \leq-E \ln \left(1-r_{T}^{1}\right)+C,
$$


where $C$ is some constant. According to (D.1), the expectations $-E \ln \left(1-r_{T}^{1}\right)$ do not converge to $+\infty$. Therefore the non-negative sums $E v_{1}+\ldots+E v_{T}$ are bounded by a constant $C_{1}$. Consequently,

$$
E \lim _{T \rightarrow \infty} \sum_{t=0}^{T} v_{t}=E \lim _{T \rightarrow \infty} \inf \sum_{t=0}^{T} v_{t} \leq \liminf \sum_{t=0}^{T} E v_{t} \leq C_{1}
$$

by virtue of the Fatou lemma. Thus, we obtain $\sum_{t=0}^{\infty} v_{t}<\infty$ a.s., hence $v_{t} \rightarrow 0$ a.s., and so $\left|R^{*}\left(s_{t-1}\right)-\zeta_{t-1}\left(s^{t-1}\right)\right| \rightarrow 0$ a.s.

\section{A.2 An Auxiliary Result}

Let $S$ be a finite set, and, for each $s \in S$, let $p(\sigma \mid s)(\sigma \in S)$ be a probability distribution on $S$ :

$$
p(\sigma \mid s) \geq 0, \sum_{\sigma} p(\sigma \mid s)=1 .
$$

For every $\sigma \in S$, let $R(\sigma, s)=\left(R_{1}(\sigma, s), \ldots, R_{k}(\sigma, s)\right)$ be a vector in the simplex $\Delta^{K}$ satisfying (A.1) and (A.2) for all $s \in S$.

Let $\rho>0$ be a number such that $R_{k}^{*}(s)>\rho, s \in S$ (see (12)). Denote by $\Delta^{K}(\rho)$ the set of those vectors $\left(b_{1}, \ldots, b_{K}\right)$ in $\Delta^{K}$ that satisfy $b_{k} \geq \rho$, $k=1, \ldots, K$. Consider the function

$$
\begin{aligned}
\Phi(s, \kappa, \mu)= & \sum_{\sigma \in S} p(\sigma \mid s) \ln \sum_{k=1}^{K} R_{k}(\sigma, s) \frac{R_{k}^{*}(s)}{R_{k}^{*}(s) \kappa+(1-\kappa) \mu_{k}} \\
& -\sum_{\sigma \in S} p(\sigma \mid s) \ln \sum_{k=1}^{K} R_{k}(\sigma, s) \frac{\mu_{k}}{R_{k}^{*}(s) \kappa+(1-\kappa) \mu_{k}}
\end{aligned}
$$

of $s \in S, \kappa \in[0,1]$ and $\mu=\left(\mu_{k}\right) \in \Delta^{K}(\rho)$.

Lemma 1 There exists a constant $L_{\rho}$ and a function $\delta_{\rho}(\gamma) \geq 0$ of $\gamma \in[0, \infty)$ satisfying the following conditions:

(a) The function $\delta(\cdot)$ is non-decreasing, and $\delta_{\rho}(\gamma)>0$ for all $\gamma>0$.

(b) For any $s \in S, \kappa \in[0,1]$ and $\mu=\left(\mu_{k}\right) \in \Delta^{K}(\rho)$, we have

$$
L_{\rho}\left|R^{*}(s)-\mu\right| \geq \Phi(s, \kappa, \mu) \geq \delta_{\rho}\left(\left|R^{*}(s)-\mu\right|\right) .
$$

Proof. It follows from (Evstigneev, Hens, and Schenk-Hoppé 2001, Lemma 1) that, for all $s \in S, \kappa \in[0,1]$ and any $\mu \in \Delta_{+}^{K}, \mu \neq R^{*}(s)$, the value of $\Phi(s, \kappa, \mu)$ is strictly positive. Fix some $\gamma_{0}>0$ for which the set $W(s, \gamma)=$ $\left\{\mu \in \Delta_{\rho}^{K}:\left|R^{*}(s)-\mu\right| \geq \gamma\right\}$ is non-empty for all $s \in S, \gamma \in\left[0, \gamma_{0}\right]$ and define

$$
\delta_{\rho}(s, \gamma)=\inf \{\Phi(s, \kappa, \mu): \kappa \in[0,1], \mu \in W(s, \gamma)\}
$$


if $\gamma \in\left[0, \gamma_{0}\right]$ and $\delta_{\rho}(s, \gamma)=\delta_{\rho}\left(s, \gamma_{0}\right)$ if $\gamma>\gamma_{0}$. Since $\Phi(s, \kappa, \mu)$ is continuous and strictly positive on the compact set $[0,1] \times W(s, \gamma)(\gamma>0)$, the function $\delta_{\rho}(s, \gamma)$ takes on strictly positive values for $\gamma>0$. Clearly this function is non-decreasing in $\gamma$. Fix some $s$, consider any $\mu \in \Delta_{\rho}^{K}$ and define $\gamma=$ $\left|R^{*}(s)-\mu\right|$. Then we have $\mu \in W(s, \gamma)$, and so

$$
\Phi(s, \kappa, \mu) \geq \delta_{\rho}(s, \gamma)=\delta_{\rho}\left(s,\left|R^{*}(s)-\mu\right|\right) .
$$

From this we can see that the sought-for function $\delta_{\rho}(\gamma)$ can be defined as

$$
\delta_{\rho}(\gamma)=\min _{s \in S} \delta_{\rho}(s, \gamma)
$$

We can write $\Phi(s, \kappa, \mu)=\Phi(s, \kappa, \mu)-\Phi\left(s, \kappa, R_{k}^{*}(s)\right)$ since the latter term is zero. The function $\Phi(s, \kappa, \mu)$ is differentiable in $\mu \in \Delta_{+}^{K}$ and its gradient $\Phi_{\mu}^{\prime}(s, \cdot, \cdot)$ is continuous, and hence bounded, on the compact set $[0,1] \times \Delta_{\rho}^{K}$. This implies the existence of the Lipschitz constant $L_{\rho}$ in (37).

\section{References}

Alchian, A. (1950): "Uncertainty, Evolution and Economic Theory," Journal of Political Economy, 58, 211-221.

Algoet, P. H., And T. M. Cover (1988): "Asymptotic Optimality and Asymptotic Equipartition Properties of Log-Optimum Investment," $A n$ nals of Probability, 16, 876-898.

Arkin, V. I., And I. V. Evstigneev (1987): Stochastic Models of Control and Economic Dynamics. Academic Press, London.

Aurell, E., R. Baviera, O. Hammarlid, M. Serva, and A. Vulpiani (2000): "Gambling and Pricing of Derivatives," Physica A, 280, 505-521.

Blume, L., And D. EAsley (1992): "Evolution and Market Behavior," Journal of Economic Theory, 58, 9-40.

(2001): "If You're So Smart, Why Aren't You Rich? Belief Selection in Complete and Incomplete Markets," manuscript, Department of Economics, Cornell University.

Breiman, L. (1961): "Optimal gambling systems for favorable games," Fourth Berkeley Symposium on Mathematical Statistics and Probability, $1,65-78$. 
Cootner, P. (1964): The Random Character of Stock Market Prices. MIT Press, Cambridge.

Evstigneev, I. V., T. Hens, and K. R. Schenk-Hoppé (2001): "Market selection of financial trading strategies: Global stability," Working Paper No. 83, Institute for Empirical Research in Economics, University of Zurich, Submitted to Mathematical Finance.

Fama, E. (1965): "The Behavior of Stock Market Prices," Journal of Business, 38, 34-105.

Hakansson, N. H., And W. T. Ziemba (1995): "Capital Growth Theory," in Handbooks in Operations Research and Management Science, Volume 9, Finance, ed. by R. A. Jarrow, V. Maksimovic, and W. T. Ziemba, chap. 3, pp. 65-86. Elsevier, Amsterdam.

Hall, P., And C. C. Heyde (1980): Martingale limit theory and its application. Academic Press, London.

Hens, T., And K. R. Schenk-Hoppé (2001): "Evolution of Portfolio Rules in Incomplete Markets," Working Paper No. 74, Institute for Empirical Research in Economics, University of Zurich, Revised Version, October 2001. Submitted to Journal of Economic Theory.

Kelly, J. L. (1956): "A New Interpretation of Information Rate," Bell System Technical Journal, 35, 917-926.

MagiLl, M., And M. QuinziI (1996): Theory of Incomplete Markets, vol. 1. MIT-Press, Cambridge.

Radner, R. (1971): "Balanced Stochastic Growth at the Maximum Rate," in Contributions to the von Neumann Growth Model, (Zeitschrift für Nationalökonomie, Suppl. 1), ed. by G. Bruckman, and W. Weber, pp. 39-62. Springer-Verlag, Vienna.

Sandroni, A. (2000): "Do Markets Favor Agents Able to Make Accurate Predictions?," Econometrica, 68, 1303-1341.

ShleIfer, A. (2000): Inefficient Markets - An Introduction to Behavioral Finance. Oxford University Press, Oxford.

Thorp, E. O. (1971): "Portfolio choice and the Kelly criterion," In Stochastic Models in Finance, W. T. Ziemba and R. G. Vickson, eds., 599-619. 